\title{
PRELIMINARY DESIGN OF AN ADAPTIVE AILERON FOR THE NEXT GENERATION REGIONAL AIRCRAFT
}

\author{
Gianluca Amendola, Ignazio Dimino, Antonio Concilio \\ Centro Italiano Ricerche Aerospaziali, Department of Smart Structures, Capua, Caserta, Italy \\ e-mail:g.amendola@cira.it; i.dimino@cira.it; a.concilio@cira.it \\ Francesco Amoroso, Rosario Pecora \\ University of Naples "Federico II", Department of Industrial Engineering, Aerospace Division, Naples, Italy \\ e-mail: f.amoroso@unina.it; rosario.pecora@unina.it
}

\begin{abstract}
Design of morphing wings at increasing TRL is common to several research programs worldwide. They are focused on the improvement of their performance that can be expressed in several ways, indeed: aerodynamic efficiency optimization, fuel consumption reduction, COx and NOx emission reduction and so on, or targeted to overcome the classical drawbacks related to the introduction of a novel technology such as system complexity increase and management of certification aspects. The Consortium for Research and Innovation in Aerospace in Quebec (CRIAQ) lunched project MD0505 that can be inserted in this crowded frame. The target of this cooperation, involving Canadian and Italian academies and a research centre, is the development of a camber "morphing aileron" integrated on an innovative full scale wing tip of the next generation regional aircraft. This paper focuses on the preliminary design and the numerical modeling of its architecture. The structural layout is, at the beginning, described in detail and furthermore, a finite element (FE) model of the entire aileron architecture is assessed and used to verify the structural integrity under prescribed operational conditions.
\end{abstract}

Keywords: morphing, actuation system, adaptive wing

\section{Introduction}

Commercial aircraft wings are typically designed for cruise operations. However, different flight phases are encountered during a standard mission; efficiency is therefore seldom optimal (Barbarino et al., 2011). The realization of lifting surfaces able to "adapt" themselves to variable operative conditions and, therefore, to match the necessity of modifying the reference configuration, may improve the current performance levels. A main feature that can be associated to a morphing structure is then for instance its potentiality to optimize the aircraft $L / D$ ratio all over the flight envelope. Several European projects, such as Clean Sky (2008) and Saristu (2012-2015) were launched in recent years to develop and assess new technologies devoted to add the structural systems with new adaptation capabilities through the use of innovative, integrated devices, demonstrating their real applicability and benefits. Aiming at those same targets, the CRIAQ Project was launched, with a specific focus on the wing trailing edge, specifically in the aileron region, (CRIAQ MDO-505, 2012). In fact, many studies (Monner et al., 1999; Bolonkin and Gilyard, 1999), demonstrated the particular effectiveness of morphing trailing edge devices located in that area. Moreover, the aileron region constitute a very delicate wing zone for several reasons. Mainly, the aileron constitutes a primary safety critical control surface whose failure is catastrophic for the entire aircraft and in addition, it must be demonstrated that no aeroelastic instability (flutter) occurs during operations. Also the reduced available space constitutes an 
important aspect which makes the morphing aileron design challenging, because it results are difficult to integrate actuators and kinematic leverage. The present paper describes the design phase of a morphing aileron prototype, ready for installation and tests in a wind tunnel. The adaptive aileron device is integrated with another complementary morphing wing system, described by Kammegne et al. (2016). The present aileron is otherwise not aimed at substituting the conventional architecture but adds new functionalities to the classical design. In fact, the aileron can still rotate rigidly around its main hinge axis while it can morph (by modifying its camber). When it is not actuated, the aileron works in the usual manner, preserving the aircraft roll control and stability (the morphing part behaves as a rigid component). In the presented application, the system works in cruise to compensate aircraft weight variations following fuel consumption. During classical manoeuver, the aileron works classically. The morphing technology can be applied also to give a better solution to the active load control on aircraft with new approaches such as active flow control (Stalewski and Sznajder, 2014), which change the flow conditions on the wing surface and, in turn, the aerodynamic loads. In the current paper, it is described how the modification of wing load distribution could be tailored to achieve wing-root bending moment alleviation as a sudden increase of aerodynamic loads occurs (gust or rapid manoeuvers). The morphing aileron is made of three-segmented ribs assembled into a finger-like architecture (Pecora et al., 2014), connected through longitudinal spars to guarantee a suitable torsional rigidity. The actuation system is completely integrated within the structural body. It includes distributed actuators the number of which is fixed according to their load-bearing capability, their force generation possibility, the allowable space and the stiffness requirements. In fact, the complete system must be able to deform while withstanding the external aerodynamic loads. These two requirements may be overcome by the use of load-bearing actuators. The kinematics allow a single degree of freedom per rib that is blocked by the actuator device. It has then the role to absorb the external load and move the system against that load. A mechanical chain converts the actuator torque into a controlled linear displacement in order to amplify the transferred force vs. a limited motion penalty. Linear motion guides are made of two main components: a stainless steel rail and a sliding element directly connected to the leverage, in turn linked to the actuator rotating shaft by means of a fork-shaped crank. The vertical force needed to move the trailing edge results by the contact between the slider and the rail. The complete system is made of commercial elements: actuators, kinematics, linear guides and all the other devices are in fact available on the market. The implemented architecture is a slight modification of the so-called quick-return mechanism (Amendola et al., 2016). In this paper, the aileron structure is sized with respect to the designated load chosen among the most critical operative ones. The working principle of the actuation system is described in detail and preliminary results of a finite element simulation are shown. Static and buckling analyses do not show any particular criticality; in other words, no plasticization arises under the limit loads, herein selected.

\section{Morphing aileron: structural layout and evaluation of loads}

The morphing aileron consists of segmented adaptive ribs based on finger-like segments enabling aileron camber morphing upon actuation. Each rib (Fig. 1) is assumed to be segmented into three consecutive blocks $(\mathrm{B} 1, \mathrm{~B} 2, \mathrm{~B} 3)$ connected to each other by means of hinges located on the airfoil camber line $(\mathrm{A}, \mathrm{B})$. Block $\mathrm{B} 1$ is rigidly connected to the rest of the wing structure through a torsion tube enabling aileron rotation for roll control. Blocks B2 and B3 are free to rotate around the hinges on the camber line, thus physically turning the camber line into an articulated chain of consecutive segments. A linking rod elements $(L)$ hinged on not adjacent blocks forces the camber line segments to rotate according to specific gear ratios. 


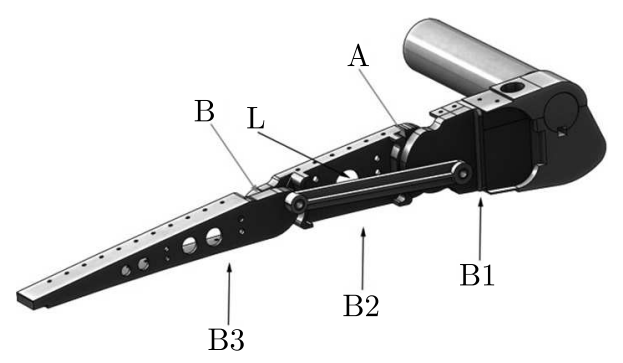

Fig. 1. Morphing rib architecture: (a) blocks and links, (b) hinges

The ribs kinematic is transferred to the overall aileron structure by means of a multi-box arrangement (Fig. 2) where the skin is hidden for clarity.

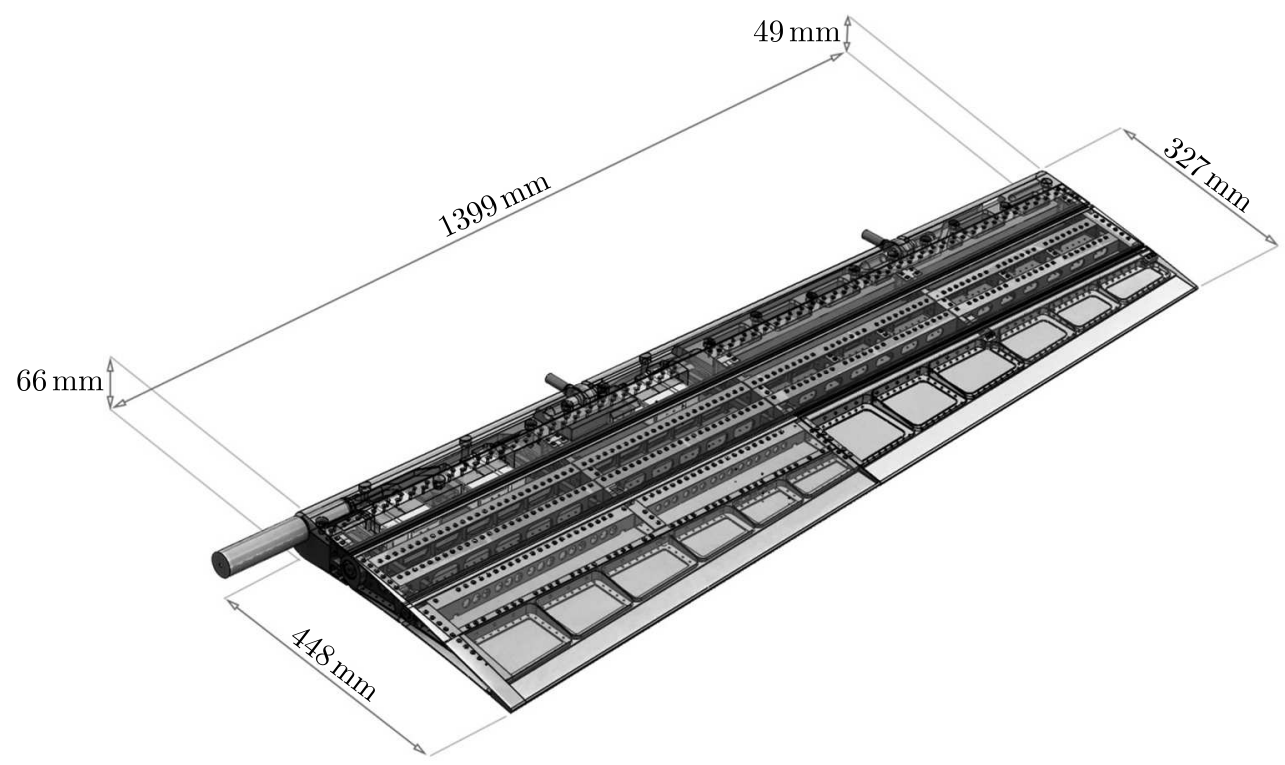

Fig. 2. Morphing aileron structure: multi-box arrangement

Referring to Fig. 3, the internal structural components are depicted, and it is also shown that the aileron is divided into one actuated and one passive segment. The internal kinematic chain actuates the first two bays while the last are considered slaved during the morphing movement.

The reference Cartesian system $S_{0}$ (Fig. 4) has been used as the datum for the load evaluation addressed by this paper; the following conceptual definition applies to $S_{0}$ :

- Origin $(O)$ at the intersection point between the Test Article (T/A) leading edge and the root rib plane;

- $X$-axis onto root rib plane, parallel to the chord of the T/A airfoil @ the root section and aft oriented;

- $Y$-axis normal to the root rib plane and oriented towards the T/A tip;

- $Z$-axis perpendicular to $X O Y$ plane and oriented upwards.

The rotation angle $\gamma$ of block B2 with respect to block B1 is determined in order to approximate target shapes by means of the articulated one-DOF mechanism described in Fig. 1. The angle $\gamma$ is represented in Fig. 5.

It is measured respect to the unmorphed chord direction and it corresponds to rigid rotation of the plain control surface comprised between $-5^{\circ}$ and $+5^{\circ}$. The VLM method has been adopted to evaluate aerodynamic pressure distribution along the aileron in correspondence to each 


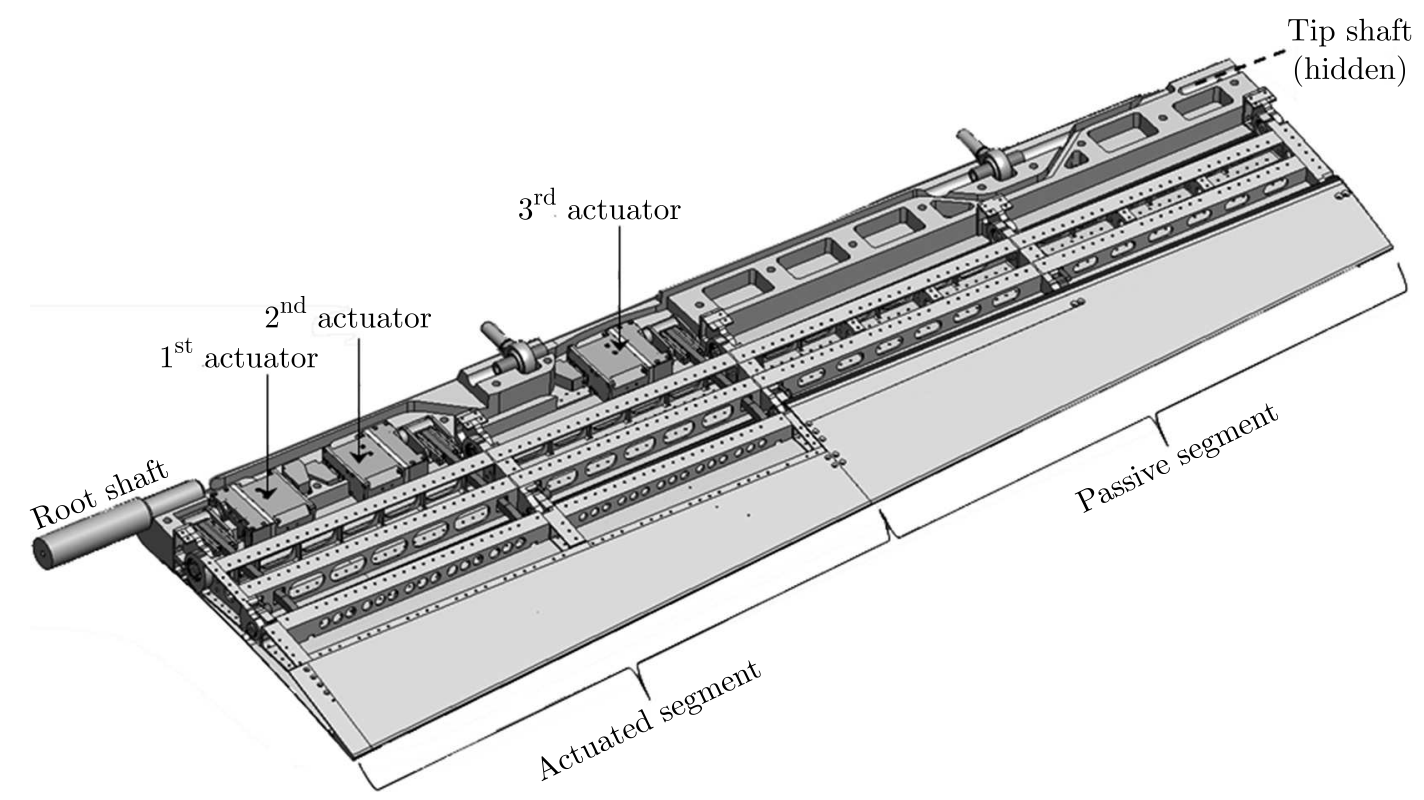

Fig. 3. CAD of the morphing aileron with an internal view to the actuation system

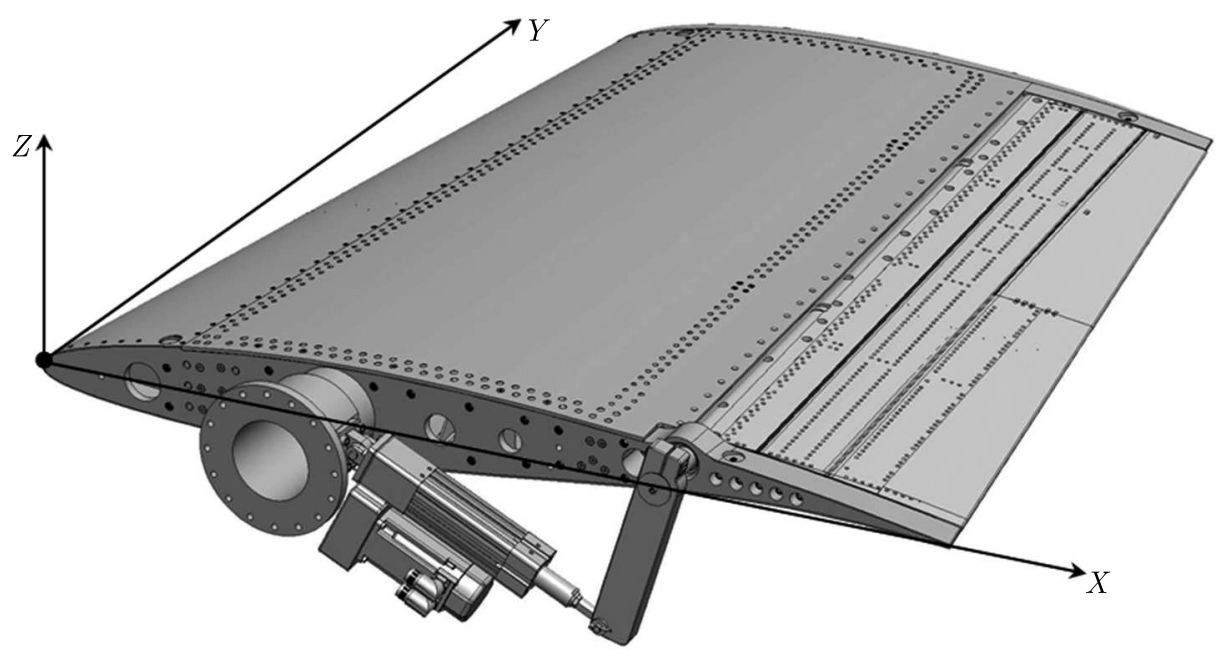

Fig. 4. CAD of the Test Article with the reference system used for aerodynamic loads
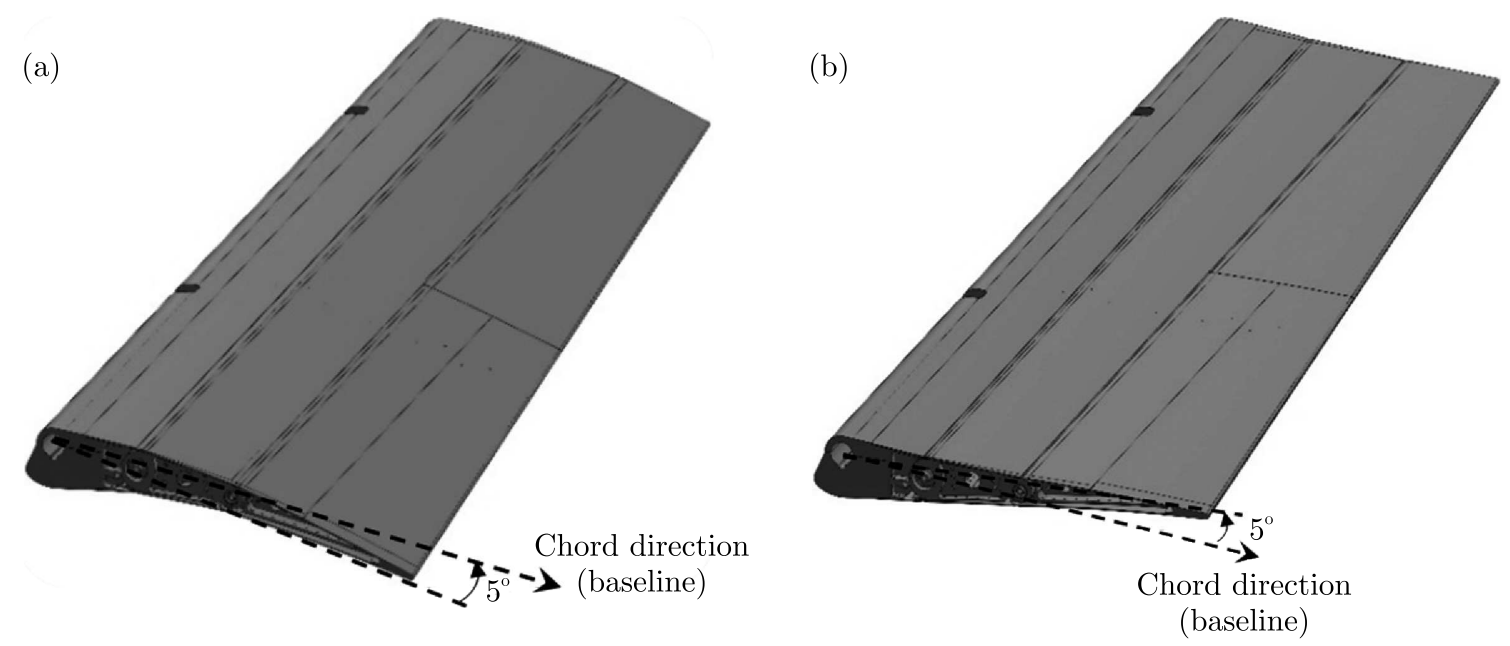

Fig. 5. Morphing aileron deflection angle $\gamma$ in morphed down and morphed up 
considered flight attitude (namely the wing angle of attack, flight altitude and speed) and aileron geometrical configuration. 3D flat-panels mesh is generated in correspondence to the outer wing segment. For each flight attitude and aileron shape, the lifting pressure $\left(P_{i}\right)$ acting along each box $\left(b_{i}\right)$ is calculated according to the following equation

$$
P_{i}=q\left(P_{0, i}+\alpha P_{\alpha, i}+\gamma P_{\gamma, i}\right)
$$

where: $q=0.5 \rho V_{\infty}^{2}$ is the dynamic pressure, $\rho$ the air density and $V_{\infty}$ the airspeed; $\alpha$ is the wing angle of attack; $P_{0, i}$ is the pressure arising on $b_{i}$ in correspondence to unit dynamic pressure at $\alpha, \gamma$ equal to zero (airfoil baseline camber effect); $P_{\alpha, i}$ is the pressure on $b_{i}$ due only to unit $\alpha$ at unit dynamic pressure (incidence effect); $P_{\gamma, i}$ is the pressure on $b_{i}$ due only to unit $\gamma$ at unit dynamic pressure (morphing effect).

Thanks to Eq. (2.1), $P_{0, i}, P_{\alpha, i}, P_{\gamma, i}$ are calculated only one time for all the boxes and then combined according to the flight attitude parameters $(\alpha, q)$ and aileron morphed shape $(\gamma)$ to be investigated. The combination of $\alpha, q, \gamma$ leading to the most significant pressure levels along aileron segments is then determined and used as the design operative condition for the structural sizing purpose. The spanwise pressure distributions on the aileron segments at the design point $\left(\alpha=2^{\circ}, q=4425 \mathrm{~N} / \mathrm{m}^{2}, \gamma=7^{\circ}\right)$ are plotted in Fig. 6 .

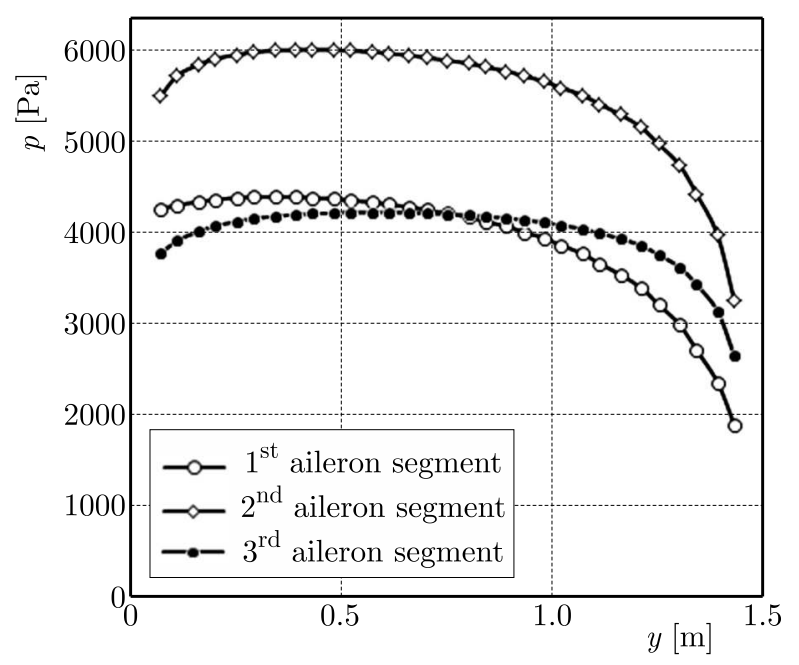

Fig. 6. Pressure distribution along aileron span

The estimated pressure distribution will be considered as the reference load for structural sizing and it will be applied to the aileron finite element model in order to asses the stress anlaysis. This constitutes the foundamental step to be done before proceeding with the manufacturing process.

\section{Actuation system}

The main target of the actuation kinematics is to develop a means of transforming the actuator motion to specific rotation of the morphing device. It must be designed to withstand the external aerodynamic loads without undergoing structural damage and at the same time to move the system to the desired morphed shape. It is based on the classical quick-return mechanism, also referred to as oscillating glyph kinematics that (Fig. 7) is widely discussed and was validated by Amendola et al. (2016).

Figure 7 shows the main structural components of the glyph kinematic system. It is composed of crank $R$ with an actuator shaft positioned at the point $O$, leverage beam $B_{L}$ connected to 


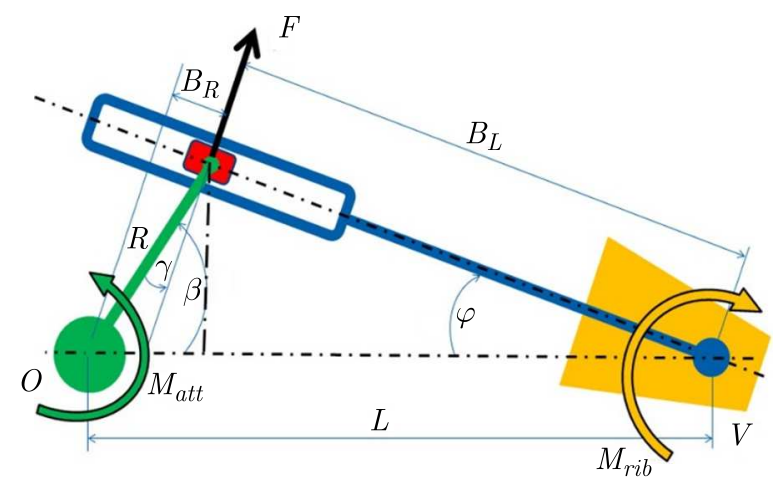

Fig. 7. Oscillating glyph kinematic scheme

aileron $B_{3}$ rib segment. The sliding element moves along its rail subjected during operation to the vertical force $F$. The actuator shaft rotation is transmitted to the structure by means of the crank $R$ and a contact force is generated by the sliding element along the linear guide. Thereby, a moment is produced that equilibrates the aerodynamic hinge moment, so that the system keeps its desired morphed shape. The mechanism is then a SDOF architecture. In the kinematic scheme, the angle $\beta$ is the actuator shaft rotation while $\varphi$ is the morphing deflection directly related to the aileron angle $\gamma$ (Fig. 5). The relation between the achieved angle and the mechanical advantage (MA), expressed as a ratio between the external load and the generated momentum, may be represented as in Fig. 8. The diagram shows that the greater rib morphing angle, the higher MA and, consequently, the actuator torque required to equilibrate the external aerodynamic moment decreases. The aileron design condition (selected as the most severe one) occurs at $\varphi=7^{\circ}$ with $\mathrm{MA}=4.2$. This peculiarity may lead to significant benefits in terms of the actuator power and weight.

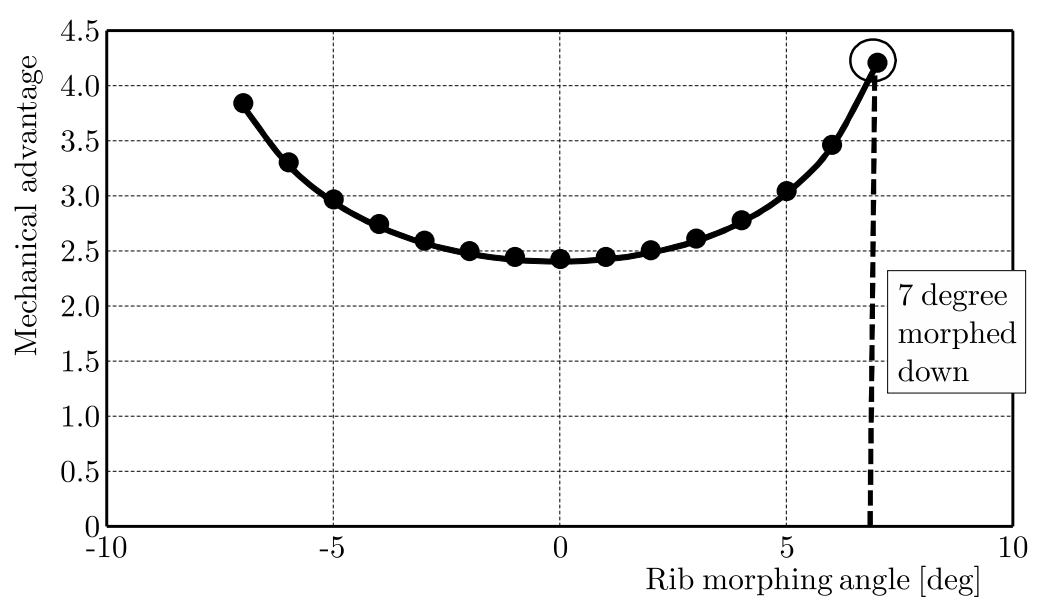

Fig. 8. MA vs. rib morphing angle

The actuator shaft rotation $\beta$ may be related to the morphing angle $\varphi$ as described by Eq. (3.1) and represented in Fig. 9

$$
\cot \varphi=\frac{L}{R \sin \beta}-\cot \beta
$$

It is evident that in the design range between $+7^{\circ}$ of morphed down and $-7^{\circ}$ of morphed up, the actuator rotation is comprised among $\pm 45^{\circ}$. The actuation system kinematics with details of the linear guides and its integration on the aileron rib are shown in Fig. 10. 


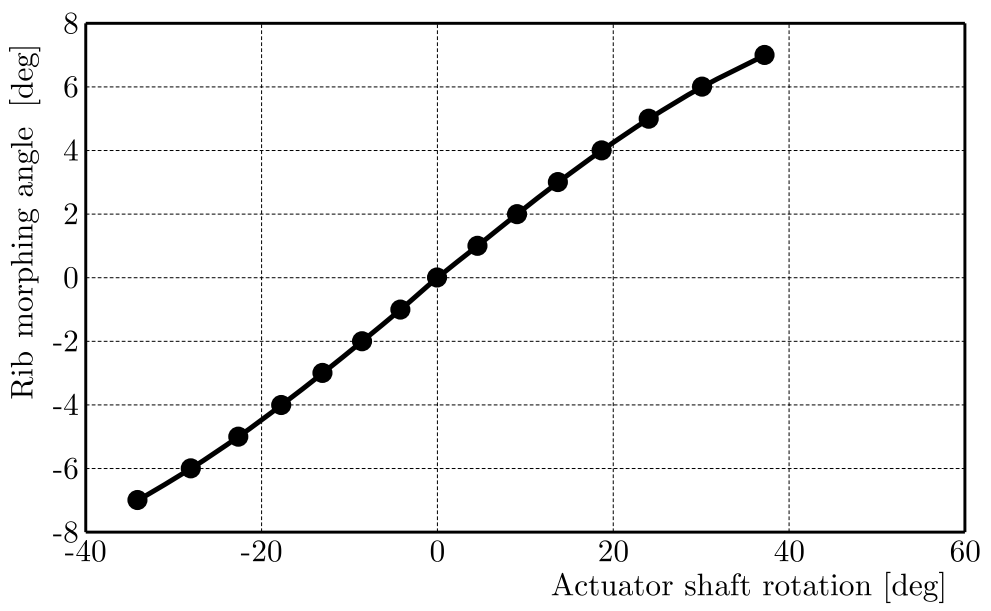

Fig. 9. Rib morphing angle vs. actuator shaft rotation
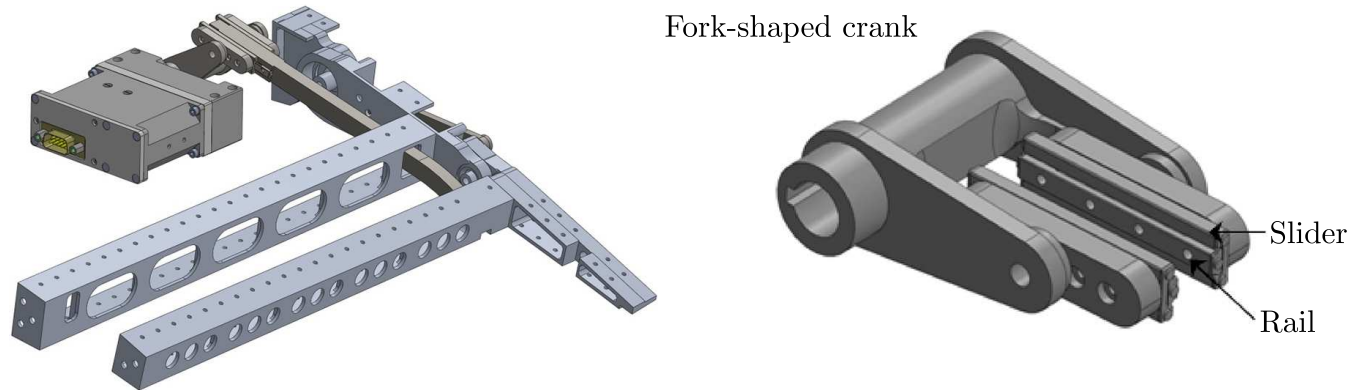

Fig. 10. Integration between the actuation system and rib (left) and details of the linear guide elements (rigth)

\section{FE validation}

In order to verify the structural robustness of the conceived morphing architectures as well as to estimate its dynamic behavior, a very refined finite element model (FEM) has been generated (Fig. 11). The model has been realized with solid finite TET10 elements both for structural components (ribs and spars) and actuation system leverages. All the hinges have been modeled by means of two-nodes CBUSH elements. Each node of the CBUSH has been rigidly connected to a representative set of nodes belonging to the structural item by means of RBE2 (Fig. 12) (MSC-Nastran).

The materials adopted for the aileron are described in Table 1 and highlighted in Figs. 13a and 13b. The aluminum components are depicted in grey while the steel components in black.

Table 1. Aileron component materials

\begin{tabular}{|c|c|c|c|l|}
\hline $\begin{array}{c}\text { Material } \\
\text { (isotropic) }\end{array}$ & $\begin{array}{c}E \\
{[\mathrm{Gpa}]}\end{array}$ & $\begin{array}{c}\rho \\
{\left[\mathrm{kg} / \mathrm{m}^{3}\right]}\end{array}$ & $\begin{array}{c}\nu \\
{[-]}\end{array}$ & Items \\
\hline \hline Harmonic steel & 210 & 7850 & 0.3 & $\begin{array}{l}\text { Beam of the actuation system, linear guide } \\
\text { features, crank and rib links }\end{array}$ \\
\hline $\mathrm{Al}$ 2024-T351 & 70 & 2768 & 0.33 & All the other items \\
\hline
\end{tabular}

The aileron model is considered constrained in correspondence to the crank exactly where the actuator shaft is located in order to prevent its rotation (clamped configuration). The following analyses have been carried out:

- Linear static analysis at the limit load

- Buckling analysis at the limit load. 


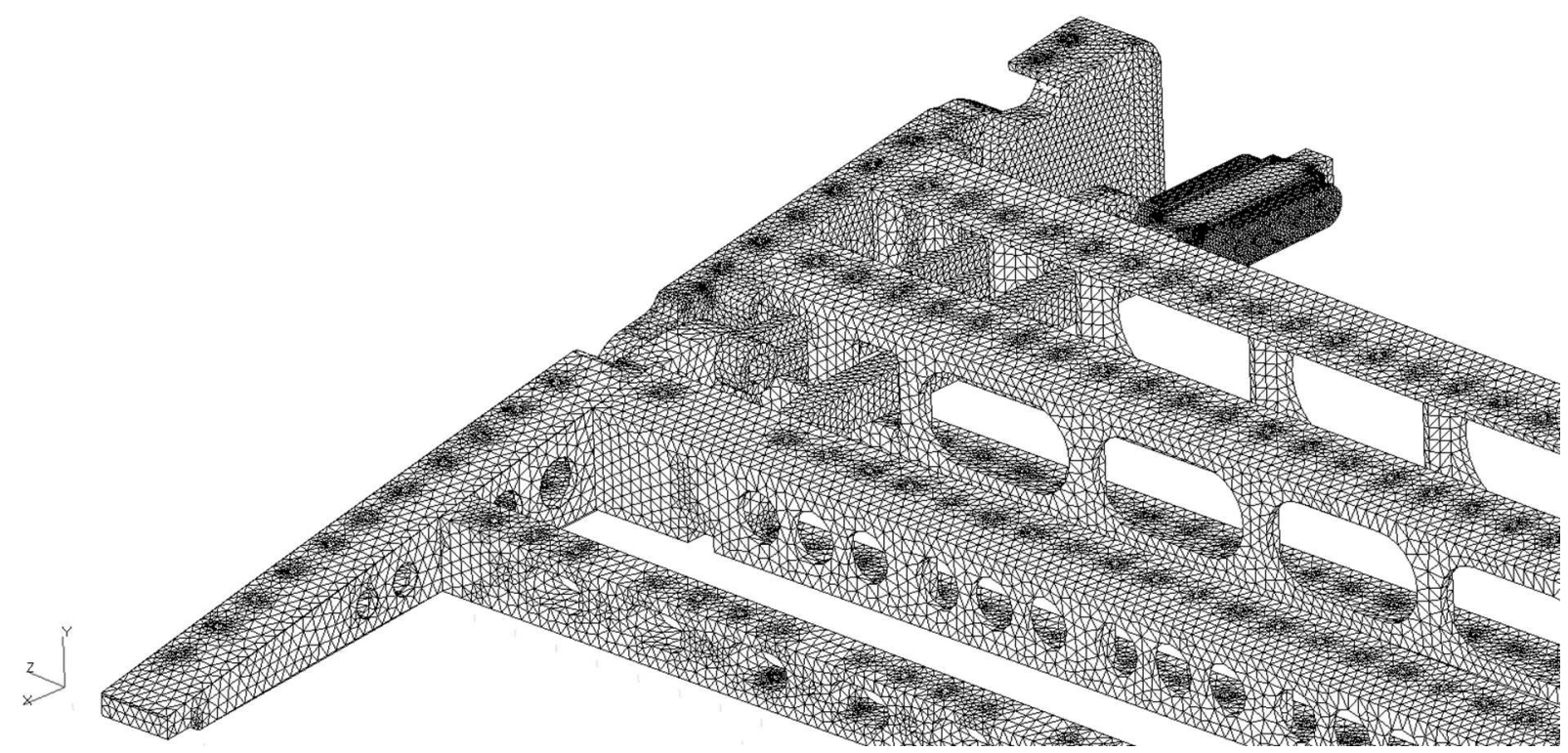

Fig. 11. Details of the aileron structure mesh

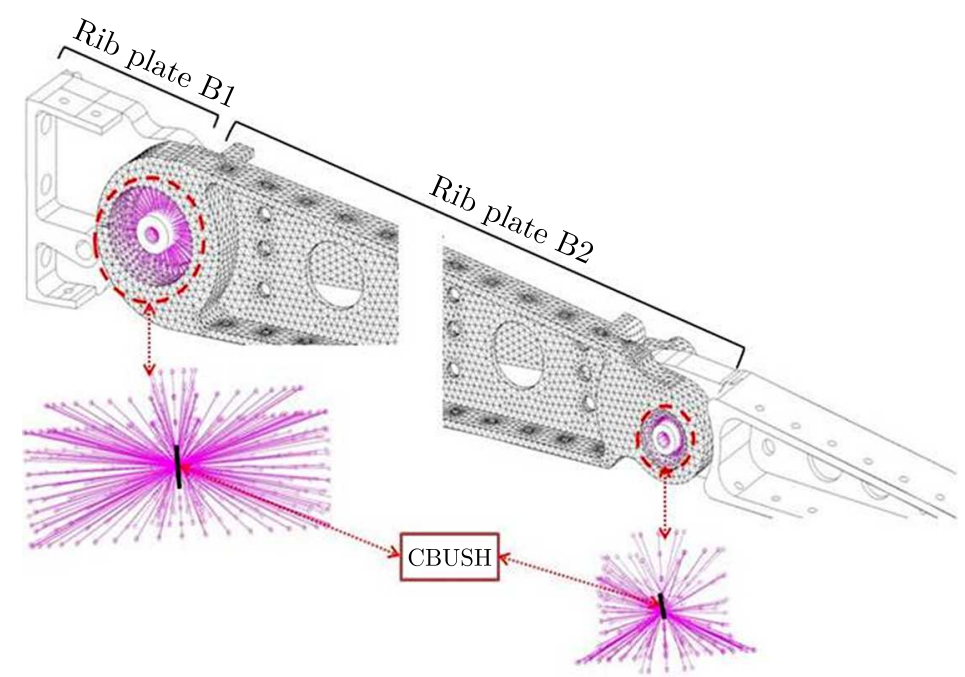

Fig. 12. Morphing aileron finite element model with details of the hinges
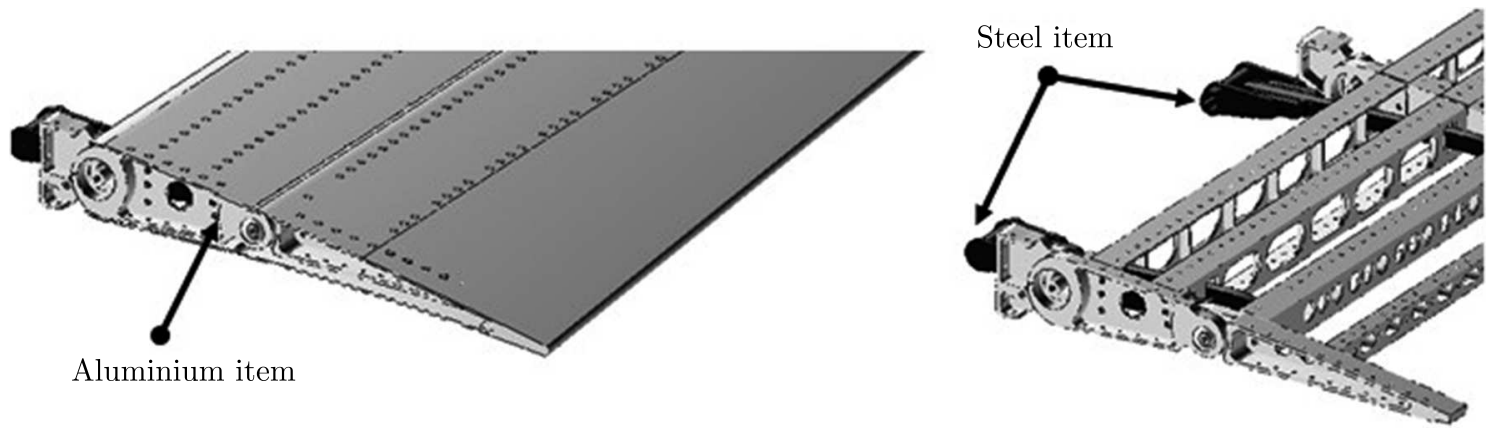

Fig. 13. Aileron materials: complete structure (a), inner structure (b)

The global magnitude of the displacements exhibited by the aileron at the limit load condition is shown. The maximum value $(21.8 \mathrm{~mm})$ is located at the trailing edge in proximity of the 1st bay (Fig. 14). The maximum von Mises stresses are detected around the rib links (257 MPa) and around hinges of the second rib $(231 \mathrm{MPa})$ and on the actuation beam $(467 \mathrm{MPa})$, resulting below the yield strength of AL 2024 alloy and steel. The described results are depicted in Figs. 14-16. 
The first buckling eigenvalue occurs at -10.391 , which means that the first critical load is more than 10 times of the applied pressure but in the opposite direction. The buckling deformation related to this eigenvalue involves rib connection links as shown in Fig. 16.

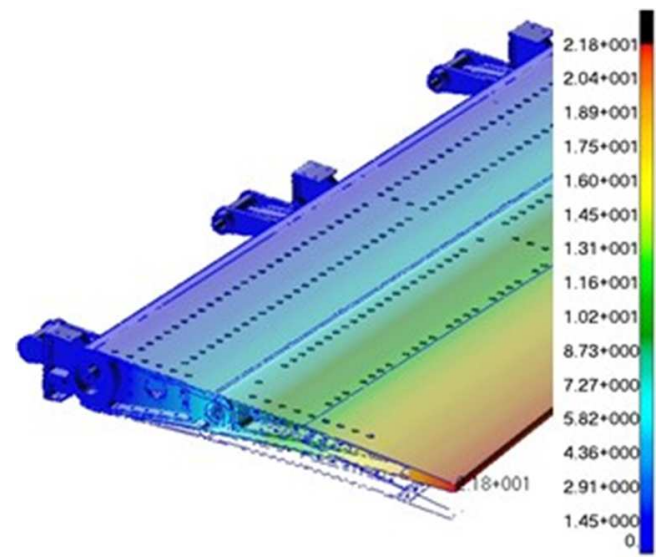

Fig. 14. Global aileron displacement distribution

Patran 20122 2 es-8at 20vut-16 165804

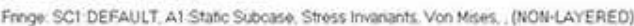
Detorm SCI DEFAULT. A1 Stabe Suocase, Oisplaceerents. Translational
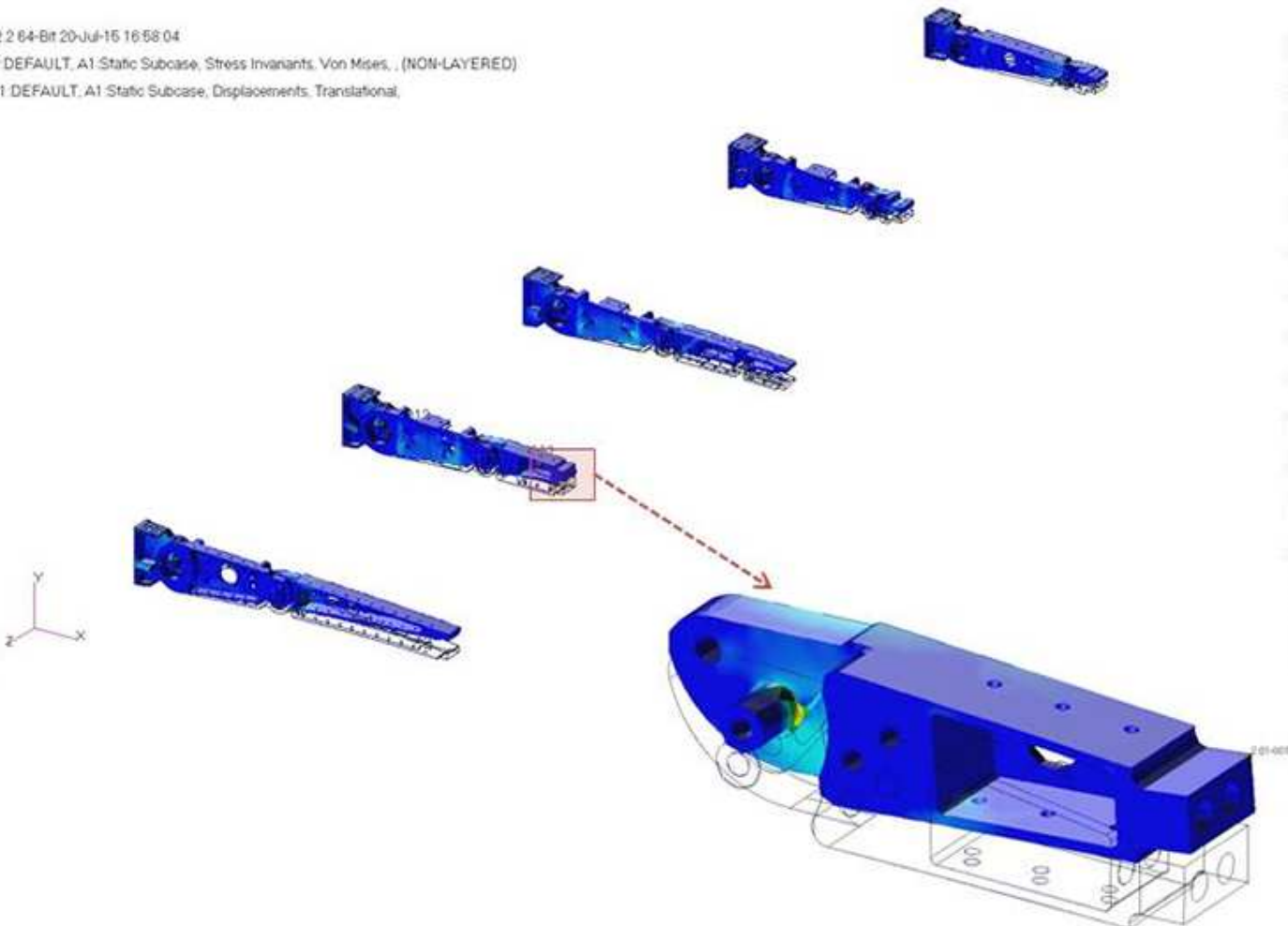

Fig. 15. Global von Mises stress distribution on the ribs

\section{Conclusions}

In this paper, the working principle of a morphing aileron actuation system is presented. The actuation mechanism is based on an oscillating glyph mechanism, combining characteristics of functionality, robustness and integrability required for adaptive structures. In particular, the study of the smart mechanical system involved functional integration of the kinematic actuation chain into a finger-like adaptive ribs architecture. The static load has been imposed to the structures and the stress results and the buckling eigenvalue have been provided. The results show that all margin of safety are positives and there are no critical points for structural safety 


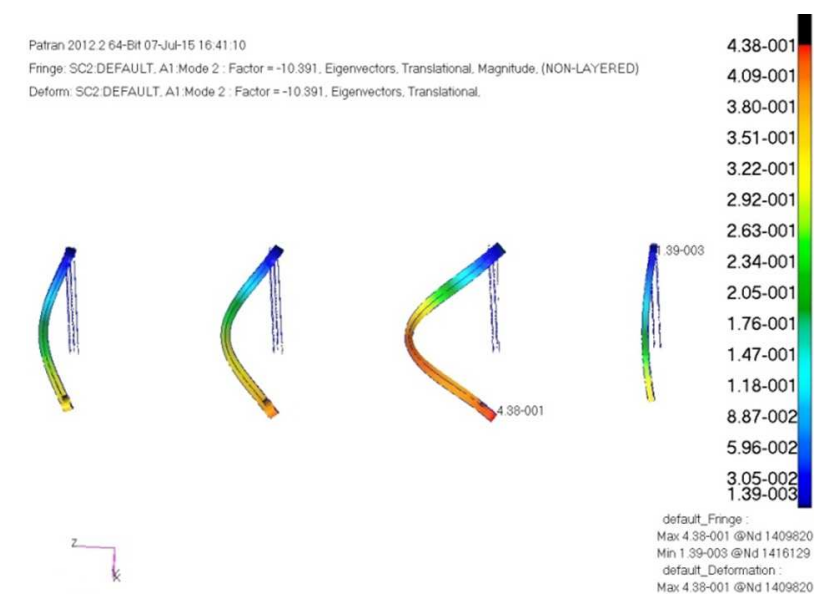

Fig. 16. First buckling deformation mode of the aileron

of the proposed morphing aileron. The next step will involve the manufacture phase where the aileron prototype will be built. Subsequently, the results herein presented will be validated by means of dedicated ground tests campaign where both static and dynamic behavior will be assessed before wind tunnel tests.

\section{References}

1. Amendola G., Magnifico M., Pecora R., Dimino I., 2016, Distrbution actuation concepts for a morphing ailreon device, The Aeronautical Journal, 120, 1231, 1365-1385, DOI: 10.1017.aer.2016.64

2. Barbarino S., Bilgen O., Ajaj R.M., Friswell M.I., Inman D.J., 2011, A review of morphing aircraft, Journal of Intelligent Material System and Structure, 22, 9, 823-877

3. Bolonkin A., Gilyard G.B., 1999, [in:] Estimated benefits of variable-geometry wing camber control for transport aircraft, NASA Technical Report

4. CRIAQ MDO-505 (2012) - Morphing Architectures and related technologies for wing efficiency improvement. Available at: http://en.etsmtl.ca/Unites-de-recherche/LARCASE/Recherche-etinnovation/Projets

5. Kammegne M.J.T., Botez M.R., Mamou M., Mebarki Y., Koreanschi A., Gabor O.S., GRIGORIE T.L., 2016, Experimental wind tunnel testing of a new multidisciplinary morphing wing model, Proceedings of the 18th International Conference on Mathematical Methods, Computational Techniques and Intelligent Systems (MAMECTIS 2016)

6. Monner H.P., Sachau D., Breitbach E., 1999, Design aspects of the elastic trailing edge for an adaptive wing, RTO AVT Specialists' Meeting on "Structural Aspects of Flexible Aircraft Control", Ottawa, Canada, 18-20 October 1999, published in RTO MP-36

7. MSC-MD/NASTRAN, 2006. Available at: http://www.mscsoftware.com/

8. Pecora R., Amoroso F., Amendola G., Concilio A., 2014, Validation of a smart structural concept for wing-flap camber morphing, Smart Structures and Systems, 14, 4, 659-679, DOI: 10.12989/sss.2014.14.4.659

9. Stalewski W., Sznajder J., 2014, Modification of aerodynamic wing loads by fluidic devices, Journal of KONES Powertrain and Transport, 21, 2, 271-278, DOI: 10.5604/12314005.1133229

10. www.cleansky.eu

11. www.ikont.eu

12. www.saristu.eu

Manuscript received February 2, 2016; accepted for print September 8, 2016 